\title{
AMENDMENTS
}

\section{Author Correction: Climate economics support for the UN climate targets}

\author{
Martin C. Hänsel D, Moritz A. Drupp (D), Daniel J. A. Johansson, Frikk Nesje, Christian Azar, Mark C. Freeman (D), \\ Ben Groom (D) and Thomas Sterner (D)
}

Correction to: Nature Climate Change https://doi.org/10.1038/s41558-020-0833-x, published online 13 July 2020.

A number of amendments are needed to the version of this Analysis originally published, none of which affect the original conclusions. We observed a slight difference in our implementation of the original DICE 2016R2 compared to Nordhaus' implementation. The difference in our implementation of the DICE 2016R2 is related to the discretization of the carbon cycle and leads to only small differences in Nordhaus' optimal base case solution. For instance, the social cost of carbon (SCC) in the year 2020 was estimated at US $\$ 39$ per ton $\mathrm{CO}_{2}$ with our discretization, but US $\$ 37$ per ton $\mathrm{CO}_{2}$ with Nordhaus' approach. The temperature increase by 2100 was estimated at $3.4^{\circ} \mathrm{C}$ with our discretization method, but $3.5^{\circ} \mathrm{C}$ with Nordhaus' approach. A minor error in the calculation of the SCC in our updated model has also been corrected. This error was present in all model runs except for the runs based on Nordhaus' discounting parameter choice and the median expert view in the fully updated model. The correction results in higher SCC for the 173 model runs based on individual expert views and also for the SCC of the median expert paths. For instance, the median expert path of our final model specification implied a SCC of US\$100 per ton $\mathrm{CO}_{2}$ before, whereas the corrected value is US\$120. Furthermore, in Extended Data Figs. 1- 3, there was a discrepancy in the EBM parameters used, while for Extended Data Fig. 2, the Carbon Cycle was not initialized correctly. In the Nordhaus baseline model in 2100, correcting these discrepancies leads to a $3 \%$ increase in atmospheric carbon concentration (Extended Data Fig. 1), $0.7^{\circ} \mathrm{C}$ higher temperature in the model with the updated EBM (Extended Data Fig. 2) and 0.7 (1.9) percentage points higher damages in the model with baseline damages (updated damages) (Extended Data Fig. 3). Subsequently, the sentence "Note that since we use a different numerical optimization solver and modelling language than does Nordhaus ${ }^{34}$, our numerical results differ slightly." has been removed from the Methods section, as what were originally thought to be solver issues have now been corrected. All errors have been amended, and all figures and numbers (for example, optimal temperature, SCC and emissions) have been updated in the main text, Extended Data, Source Data and Supplementary Information where necessary; the original and corrected figures are provided as Supplementary Information to this notice. All results related to the optimal solutions for the atmospheric temperature, carbon concentrations and emissions obtained using our updated version of DICE, remain unaffected. The headline conclusions are unchanged.

\section{Additional information}

Supplementary information The online version contains supplementary material available at https://doi.org/10.1038/s41558-021-01021-w.

Published online: 31 March 2021

https://doi.org/10.1038/s41558-021-01021-w

( ) The Author(s), under exclusive licence to Springer Nature Limited 2021

\section{Publisher Correction: Reply to: Eurasian cooling in response to Arctic sea-ice loss is not proved by maximum covariance analysis}

\author{
Masato Mori (D), Yu Kosaka (D), Masahiro Watanabe (D), Bunmei Taguchi (D), Hisashi Nakamura and Masahide Kimoto \\ Correction to: Nature Climate Change https://doi.org/10.1038/s41558-020-00983-7, published online 2 February 2021.
}

In the version of this Matters Arising Reply originally published, in the 'Replying to' line, the year '2010' was incorrect and should have been '2021'. The online versions of this Matters Arising Reply have been corrected.

Published online: 16 February 2021

https://doi.org/10.1038/s41558-021-01003-y

(c) The Author(s), under exclusive licence to Springer Nature Limited 2021 